Wie präsentiert man 175 Jahre Medizinstudium? Der Beitrag, aus dem die heutigen Auszüge stammen, nimmt den Leser mit auf einen Rundgang durch die Ausstellung «Vom Grünschnabel zum Weisskittel» und damit auch auf einen Rundgang durch verschiedenste Bereiche des studentischen Lebens.

\title{
Zwischen Kanzlei, Hörsaal und Kneipe: Ein Rundgang durch die Ausstellung*
}

Wiebke Schweer

\footnotetext{
* Auszug aus dem gleichnamigen Beitrag der Autorin in: Iris Ritzmann, Wiebke Schweer, Eberhard Wolff (Hrsg.). Innenansichten einer Ärzteschmiede. Lehren, lernen und leben - aus der Geschichte des Zürcher Medizinstudiums. Zürich: Chronos; 2008. 240 S., 80 teils farbige Abbildungen. Fr. 34.-, $€ 20.60$ ISBN 978-3-0340-0909-6. Quellenverweise im Originalartikel.
}

Korrespondenz:

Wiebke Schweer M.A.

Universität Zürich

Medizinhistorisches Institut

Hirschengraben 82

CH-8001 Zürich

Tel. 0446340005

wiebke.schweer@mhiz.uzh.ch
Die Besucherinnen und Besucher der Ausstellung begleiten die Grünschnäbel durch ihr Studium, sitzen mit ihnen im Hörsaal, pauken mit ihnen am Schreibtisch und zittern in der Prüfung. Sie schauen ihren Professoren im Kabinett bei der Vorbereitung für ihre Vorlesungen über die Schulter und können dann in den buntbebilderten Mitschriften nachlesen, was die Studierenden davon verstanden haben.

Bis die Universität mit dem Diplom in der Hand verlassen werden konnte, stand erst einmal ein wahrer Vorlesungsmarathon an. Für den Studenten Carl Meyer ging es in seinem ersten Semester zum Beispiel am Dienstagmorgen um acht Uhr mit «Differentialrechnung mit Übungen» los. Sein Tag an der Universität endete dann um 19 Uhr mit der «Allgemeinen Botanik». Kein Wunder, dass die Studierenden sich manchmal fühlten, als ob ihnen das Wissen nur so eingetrichtert wurde, wie eine Karikatur aus einer der studentischen Satirezeitungen zeigt - im Sommersemester 1889 drückte Carl Meyer teilweise 51 Stunden pro Woche die Hörsaalbank.

Hier kann auch der Ausstellungsbesucher Platz nehmen, für ihn steht Ophthalmologie auf dem Stundenplan. Kein Wunder also, dass ihn gleich mehrere Augen fest im Blick haben. Es sind originalgetreu gemalte Bilder auf überlebensgrossen Lehrtafeln, die im Hörsaal zu Anschauungszwecken hingen. Fein abgezeichnet finden sich die Motive in den Mitschriften der Studierenden wieder, die zum Nachlesen ausliegen - die älteste aus dem Jahr der Universitätsgründung, die jüngste von 1941. Als die Bilder laufen lernten, wurden auch von den Professoren Filme produziert und eingesetzt. So flimmert ein Lehrfilm aus den 1930er Jahren über die Leinwand, der Lähmungserscheinungen von Augen zeigt.

Die Exponate dokumentieren, wie Lehrinhalte im Laufe der vergangenen 175 Jahre in Bilder umgesetzt wurden, um das Lernen zu erleichtern. Die Ausstellung greift zum gleichen Trick und lässt Personen, Geschichten und Gegenstände mit Modellen, Hörstationen, Computersimulationen und Filmen lebendig werden.

Die Studierenden machten sich, wenn sie nicht gerade eifrig mitschrieben und auswendig lernten, ihr eigenes Bild von Lehre und Lehrenden - zumindest mit dem Zeichenstift. Im «Studio», dem Raum in der Ausstellung, der ganz dem

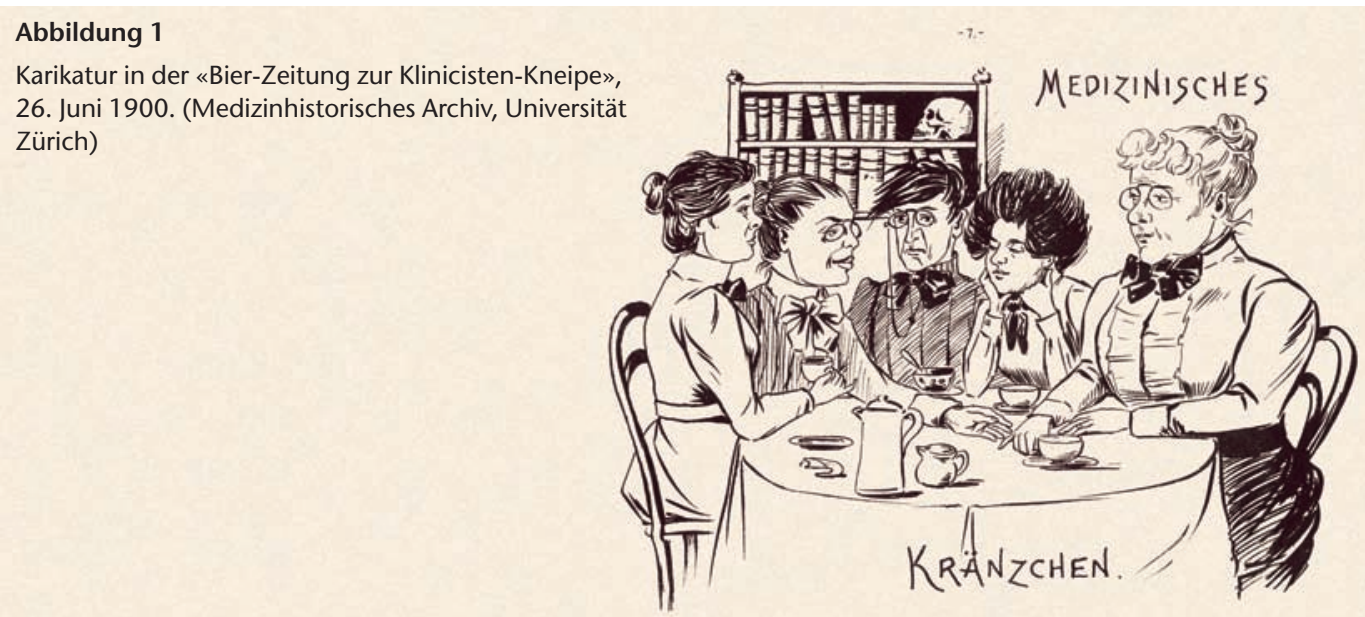


Nachwuchs gewidmet ist, kann der Besucher in den sogenannten «Bierzeitungen» lesen, in denen die Studierenden Karikaturen, Spottverse, Geschichten und Gerüchte zu Professoren und Kommilitonen verbreiteten. Neben den Lehrern bekamen dabei besonders die Studentinnen ihr Fett weg. In den Anfangsjahren des Frauenstudiums konnten es die Männer offensichtlich nicht verwinden, dass die Frauen neben ihnen nicht mehr nur hübsche Anhängsel an ihrer Seite waren, sondern Kommilitoninnen, dazu noch überaus fleissige. Zahlreiche Karikaturen in den Bierzeitungen zeigen sie als hässliche Jungfern, zum Beispiel beim Kaffeekränzchen.

\section{Abbildung 2}

Kolleghefte des Zürcher Medizinstudenten Friedrich Goll über die «Physiologie des Menschen» im Sommersemester 1852. Die Kolleghefte lückenlos zu führen füllte einen Grossteil der Studienzeit. Die aufwendige Bindung von Golls Kollegheften ist ungewöhnlich und erfolgte wahrscheinlich nachträglich. (Medizinhistorisches Archiv, Universität Zürich. Fotografie: Lutz Hartmann, Zürich)

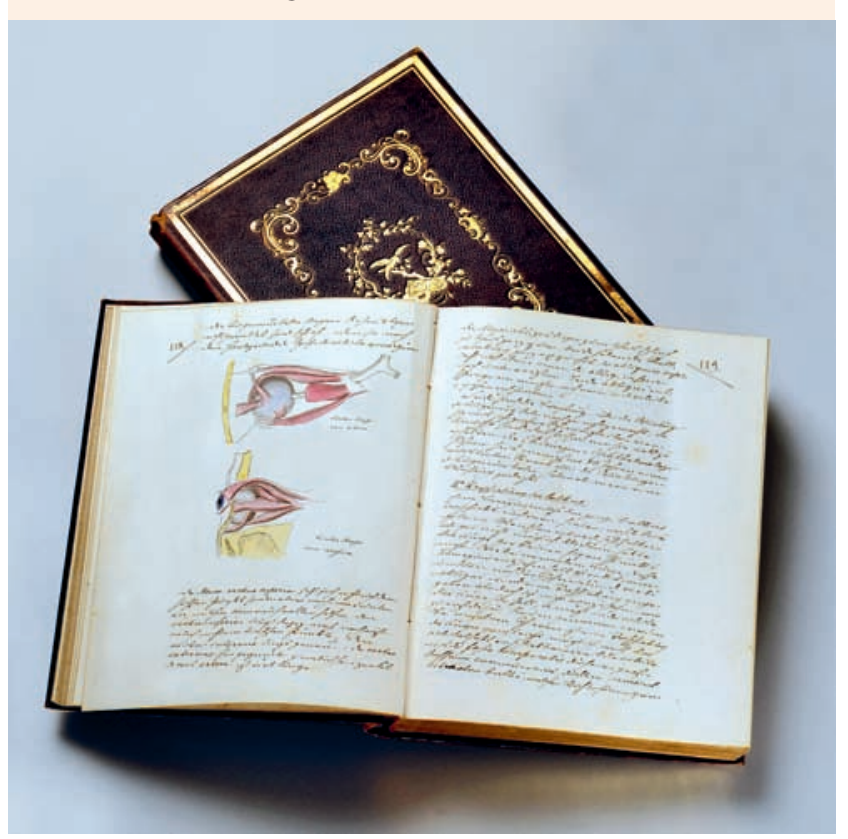

In der nächsten Folge: «Debatten über das ideale Medizinstudium in Zürich und der ganzen Schweiz».

\section{Abbildung 3}

Gemalte Lehrtafel «Hyperämie des Auges», die drei verschiedene Augenrötungen darstellt. Die Lehrtafel entstand vermutlich unter Anleitung des Zürcher Ophthalmologieprofessors Alfred Vogt und wurde um $1930 \mathrm{im}$ Unterricht eingesetzt. (Medizinhistorisches Archiv, Universität Zürich. Fotografie: Lutz Hartmann, Zürich)

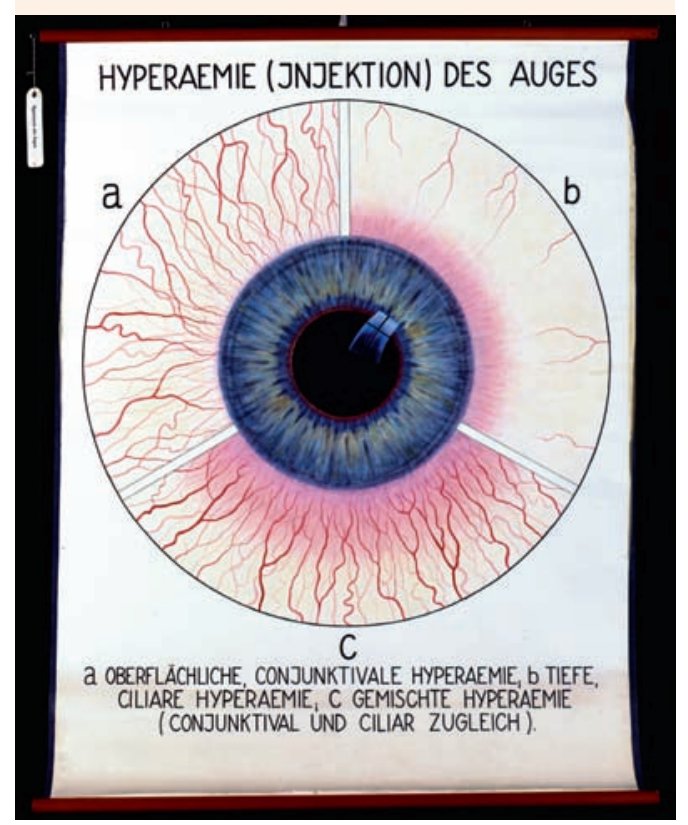

